\title{
Uso de cetamina pela via cólon-retal para contenção química de jiboias Boa constrictor Linnaeus, 1758 com teste de influência da droga sobre o sistema renal ${ }^{1}$
}

\author{
Tatiale O. Rodrigues ${ }^{2 *}$, André L.Q. Santos ${ }^{2,3}$, Paulo V.R. Pereira ${ }^{4}$, \\ Sthéfani E. Siqueira ${ }^{2}$, Thaís C.S. Rodrigues ${ }^{2}$ e Ednaldo C. Guimarães ${ }^{5}$
}

\begin{abstract}
Rodrigues T.O., Santos A.L.Q., Pereira P.V.R., Siqueira S.E., Rodrigues T.C.S. \& Guimarães E.C. 2018. [Use of ketamine by colon-rectal route for chemical restraint in Boa constrictor Linnaeus, 1758 with test of influence of the drug on renal system.] Uso de cetamina pela via cólon-retal para contenção química de jiboias Boa constrictor Linnaeus, 1758 com teste de influência da droga sobre o sistema renal. Pesquisa Veterinária Brasileira 38(2):328-334. Laboratório de Pesquisa em Animais Silvestres, Universidade Federal de Uberlândia, Avenida Piauí s/n, Campus Umuarama, Uberlândia, MG 38405-314, Brazil. E-mail: tatialerodrigues@yahoo.com.br

It is necessary to deal with Boa constrictor snakes because they are frequently treated in wild and exotic animal clinics and the knowledge about the required procedures in the treatment of the possible affections becomes imperative. When the choice for chemical restraint or sedation for surgical procedures is made, one of the drugs used in snakes is ketamine. We believed it was necessary to look for an alternative route of drug administration as effective as the regular ones, but with minimum risks and less adverse effects in its execution. Therefore thirteen snakes were submitted to the administration of $70 \mathrm{mg} / \mathrm{kg}$ of ketamine hydrochloride, with an urethral tube, through the cloaca into the colon-rectum. After this, they were evaluated during the $0,10,20,30,40,50,60,70,80,90$ and 120 next minutes from the administration time using the following parameters: heart rate, muscle relaxation and mobility, handling or restraint resistance and righting reflex. Blood samples were collected from each snake by cervical paravertebral venous sinus punction, before the drug administration, on the next day and nine days after. Serum concentrations of calcium, phosphorus and uric acid were measured in order to check the renal biochemical profile and the possibility of influence of the drug on this system. It was possible to provoke chemical restraint in Boa constrictor snakes, with ketamine hydrochloride administered by the colon-rectal route.
\end{abstract}

INDEX TERMS: Colon-rectal route, ectothermic animals, anesthesia, renal function, enema, snakes, Boa constrictor, ketamine, anesthesiology.

\footnotetext{
${ }^{1}$ Recebido em 12 de junho de 2017.

Aceito para publicação em 26 de junho de 2017.

${ }^{2}$ Laboratório de Pesquisa em Animais Silvestres (LAPAS), Universidade Federal de Uberlândia, Avenida Piauí s/n, Campus Umuarama, Uberlândia, MG 38405-314, Brasil. *Autor para correspondência: tatialerodrigues@yahoo.com.br

${ }^{3}$ Departamento de Anatomia dos Animais Domésticos, Universidade Federal de Uberlândia, Avenida Mato Grosso 3289, Bloco 2-S, Campus Umuarama, Uberlândia, MG 38405-314, Brasil.

${ }^{4}$ Laboratório Escola, Faculdade Patos de Minas (FPM), Rua Major Gote 1408, Centro, Patos de Minas, MG 38700-000, Brasil.

${ }^{5}$ Faculdade de Matemática, Universidade Federal de Uberlândia, Campus Santa Mônica, Av. João Naves de Avila, 2121, Uberlândia, MG 38408-100, Brasil.
}

RESUMO.- Por serem frequentes na clínica de animais silvestres, faz-se necessária a manipulação de jiboias e para isso, indispensável o conhecimento sobre as manobras precisas para o tratamento das possíveis afecções. Quando se opta pela contenção química, ou pela realização de procedimentos cirúrgicos, um dos fármacos utilizados na anestesia de serpentes é a cetamina. Viu-se a necessidade de buscar uma via alternativa, semelhante em eficácia às tradicionais para a contenção química, porém que minimizasse os riscos e efeitos adversos encontrados na sua execução. 0 presente trabalho sugere que a via retal seja esta alternativa, por isso, treze jiboias foram submetidas à administração de $70 \mathrm{mg} / \mathrm{kg}$ de cloridrato de cetamina, com sonda uretral, através da cloaca até o cólon-reto. Foram avaliados, nos 
tempos $0,10,20,30,40,50,60,70,80,90$ e 120 minutos, a partir da administração do fármaco, os seguintes parâmetros: frequência cardíaca, relaxamento muscular e mobilidade, resistência à contenção ou manipulação e reação postural de endireitamento. Foi realizada coleta de $0,5 \mathrm{~mL}$ de sangue por punção do seio venoso paravertebral cervical, antes da administração do fármaco, no dia seguinte e após nove dias. Foram dosadas as concentrações plasmáticas de cálcio, fósforo e ácido úrico de todos os exemplares a fim de verificar o perfil bioquímico renal e avaliar a influência do fármaco neste sistema. Não foram observadas alterações bioquímicas plasmáticas durante o período de avaliação. Foi possível promover a contenção química das jiboias Boa constrictor, utilizando cloridrato de cetamina pela via cólon-retal.

TERMOS DE INDEXAÇÃO: Via cólon-retal, répteis, anestesia, função renal, enema, serpentes, jiboia, Boa constrictor, cetamina, anestesologia.

\section{INTRODUÇÃO}

As jiboias da espécie Boa constrictor Linnaeus, 1758, são répteis pertencentes à família Boidae, da Ordem Squamata e Subordem Ophidia, são animais ectotérmicos, ou seja, sua temperatura corporal varia de acordo com a temperatura do ambiente (Funk 2006). Por serem animais frequentes na clínica de animais silvestres e pertencentes à fauna brasileira encontrados em vida livre, ou mantidos em cativeiro, inclusive criados como animais de estimação, faz-se necessária a manipulação desses animais e para isso, indispensável o conhecimento sobre as características da espécie e as manobras precisas para o tratamento das suas possíveis afecções (Bennett 1991, Carregaro et al. 2009).

Procedimentos ambulatoriais e exame clínico detalhado não são facilmente realizados em serpentes apenas com a contenção física (Bennett 1991, Valente et al. 2013), assim, quando opta-se pela contenção química, ou pela realização de procedimentos cirúrgicos, um dos fármacos utilizados na sedação de serpentes é a cetamina (Bennett 1991, Malley 1997, Schumacher \& Yelen 2006, Carregaro et al. 2009).

A via de administração é um fator a ser considerado na escolha do protocolo anestésico, levando em consideração o estresse que a administração pode causar aos animais, neste caso as vias enterais podem ser consideradas menos invasivas e dolorosas. As vias tradicionalmente usadas na administração de fármacos nas serpentes são as vias oral e intramuscular (Valente et al. 2013). Manipular a boca de uma serpente é um agravante ao risco de mordida, exigindo-se grande habilidade e cuidado, tornando mais difícil e desconfortável também para o animal a administração de fármacos oralmente. Uma alternativa é a mistura do medicamento com o alimento, o que também é complicado pela espécie em questão alimentar-se exclusivamente de presas e apesar de possível para outros fármacos, não se aplicaria a anestésicos (Mitchell 2006, Valente et al. 2013). A distribuição da musculatura pelo corpo das serpentes faz-se de forma peculiar, sendo que a única musculatura recomendada para administração de fármacos é a paravertebral, que não suporta a administração de maiores volumes e encontra-se ainda mais reduzida em animais debilitados. Tais obstáculos dificultam a administração de medicamentos via intramuscular em serpentes, além disso, essa ainda é considerada uma via invasiva e dolorosa. A área de aplicação de fármacos pelas vias parenterais em serpentes torna-se ainda menor quando se considera a administração apenas no terço anterior do corpo, baseado na influência do sistema porta-renal no metabolismo das substâncias (Bennett 1991, Mitchell 2006, Valente et al. 2013, Grego et al. 2014). Para a realização da via endovenosa em serpentes recomenda-se uma pequena incisão de pele e a via intraóssea não é possível de ser acessada (Mitchell 2006, Valente et al. 2013).

Com isso, viu-se a necessidade de buscar uma via alternativa, semelhante em eficácia às tradicionais, porém que minimizasse os riscos e efeitos adversos encontrados na sua administração. Baseando-se no que traz a literatura sobre vias alternativas de administração em diferentes espécies (Hanna et al. 1988, Podell et al. 1998, Steinman et al. 2000, Berkó et al. 2002, Papich 2003, Zhu et al. 2005, Wimsatt et al. 2008), o presente trabalho sugere que a via retal seja uma opção, acreditando ser uma via menos invasiva que gere menor estresse (Zempsky 1997) e que possa resultar em leve sedação, proporcionando uma manipulação das serpentes que seja tranquila para o profissional e igualmente para o animal.

Porque as serpentes, assim como os demais répteis apresentam o sistema porta renal, evita-se aplicação de fármacos na porção posterior do corpo presumindo-se o risco de fármacos com potencial nefrotóxico causarem lesão renal (Grego et al. 2014), por este ser um provável obstáculo da via proposta, houve a necessidade de avaliar também a influência do fármaco sobre o sistema renal.

Também é importante determinar protocolos seguros e eficazes de contenção química de serpentes (Bennett 1991, Valente et al. 2013). Como a cetamina é utilizada há muitos anos em serpentes e é conhecida como um anestésico seguro (Glenn et al. 1972, Hill \& Mackessy 1997, Font \& Schwartz 1989), foi escolhida para este teste.

A cetamina é um dos fármacos que têm a função de dissociar o córtex cerebral, mantendo os reflexos protetores. A droga é capaz de produzir analgesia profunda com catalepsia, além de ter conhecida ação simpatomimética (Massone 2011), seus metabólitos são excretados pelos rins (Kohrs \& Durieux 1998). 0 pKa da cetamina é de 7,5 e o peso molecular de 238 Dalton, com alta lipossolubilidade, é uma 2-(o-clorofenil)-2-(metilamino)cicloexanona, sintetizada por Stevens em 1963, cujo isômero levo-rotatório é mais potente do que o dextro-rotatório, além de ter biodisponibilidade de 93\%, meia-vida plasmática de 186 minutos e meia-vida de distribuição de 11 a 16 minutos. É um agente que atua em diversos receptores do sistema nervoso central e bloqueia de maneira não competitiva os receptores NMDA (Oliveira et al. 2004). Estas características vão influenciar na sua absorção, metabolização, ação e excreção; assim sendo, vão ditar se a cetamina pode ser efetiva quando administrada pela via cólon-retal de jiboias.

Ainda que, até então, não tenha sido relatado ou evidenciado potencial nefrotóxico da cetamina, é importante lembrar que esta realidade é conhecida a partir do uso convencional do fármaco, que ao ser administrado por outras vias é metabolizado pelo fígado antes de passar pelo sistema renal (Kohrs \& Durieux 1998). Questiona-se, assim como para outros fármacos, se a ausência dessa passagem pelo fígado devido ao desvio do sistema porta renal (Wright 2006), que pode ou não acontecer na forma de administração proposta, seria um obstáculo ao uso da via cólon-retal para administração de cetamina em serpentes.

Trabalhos semelhantes não foram encontrados na literatura consultada, o que leva a concluir que este seria o primeiro a testar a eficácia deste método. Mesmo em outras espécies animais, 
faltam estudos que apontem as vantagens e desvantagens do uso da via retal para administração de fármacos.

Objetiva-se testar a eficácia da administração de cloridrato de cetamina pela via cólon-retal em jiboias (Boa constrictor).

\section{MATERIAL E MÉTODOS}

Aspectos éticos. 0 experimento foi conduzido no Laboratório de Ensino e Pesquisa em Animais Silvestres (LAPAS) e foi aprovado pela Comissão de Ética na Utilização de Animais (CEUA) da Universidade Federal de Uberlândia (UFU), protocolo no138/15; com licença no Sistema de Autorização e Informação em Biodiversidade (SISBIO) concedida pelo Instituto Chico Mendes de Conservação da Biodiversidade (ICMBIO) e Ministério do Meio Ambiente (MMA) sob o protocolo nํ 52002-1.

Desenho experimental. Treze jiboias (seis fêmeas e sete machos) da espécie Boa constrictor foram mantidas em jejum alimentar sem restrição hídrica durante 7 dias. Após este período foram pesadas individualmente e a média de peso das serpentes utilizadas no experimento foi de $1,74 \pm 0,65 \mathrm{~kg}$. As jiboias foram contidas fisicamente para coleta de $0,5 \mathrm{~mL}$ de sangue através de punção do seio venoso paravertebral cervical, utilizando seringa de $5 \mathrm{~mL}\left(\mathrm{SR}^{\circledR}\right.$ - Luer Lock) com heparina sódica (Hemofol ${ }^{\circledR}$ - Cristália) acopladas a agulhas de diâmetro 1,3x0,45mm - 26 G1/2 (Descarpack ${ }^{\circledR}$ ). Procedeu-se a aferição da temperatura retal, utilizando o termômetro do monitor multiparamétrico (IMFTEC, Modelo: Guthen 1000). Em seguida, foi administrado $70 \mathrm{mg} / \mathrm{kg}$ do anestésico cloridrato de cetamina (Dopalen ${ }^{\circledR}$ - Ceva Brasil), com seringas de 1 ou $3 \mathrm{~mL}\left(\mathrm{SR}^{\circledR}\right.$ - Luer Lock), a depender do volume a ser administrado, acopladas a sonda uretral de número 06, 08 ou 10, a depender do tamanho do animal e devidamente lubrificada com gel a base de água, introduzida através da cloaca até o reto. As serpentes foram mantidas durante todo o procedimento em sala com monitoramento constante de temperatura com auxílio de termo-higrômetro digital (Incoterm/Modelo CE/ABS).

Avaliação dos animais. Então, foram avaliados, nos tempos $0,10,20,30,40,50,60,70,80,90$ e 120 minutos, sendo o tempo 0 o momento imediatamente anterior à administração do fármaco, os seguintes parâmetros:

a) frequência cardíaca, com auxílio de doppler vascular ultrassônico (MEDPEJ, Modelo: DV-2001), os batimentos eram contados durante um minuto;

b) relaxamento muscular e mobilidade, sendo atribuído escore (1) para animais ativos com postura corporal e mobilidade altas como no período pré anestésico, (2) intermediário e (3)completo relaxamento e ausência de mobilidade;

c) facilidade de contenção física/manipulação, atribuindo-se escore (1) para animais que apresentaram resistência à contenção física e manipulação semelhantes ao observado no período pré anestésico, (2) animais que apresentaram resistência intermediária, (3) não resistência;

d) reação postural de endireitamento, que foi o tempo (em segundos) que o animal levou para retomar o posicionamento natural após ser colocado em decúbito dorsal.
Após o último tempo de avaliação, a temperatura retal foi aferida novamente e os animais foram realocados em suas caixas. 24 horas depois, os animais foram novamente contidos fisicamente para coleta de $0,5 \mathrm{~mL}$ de sangue por punção do seio venoso paravertebral cervical. Depois, foram alimentados e retornaram ao manejo de rotina. Após 9 dias, as serpentes foram contidas para a última coleta de sangue.

Análises bioquímicas. As amostras de sangue foram armazenadas em tubo para coleta de sangue a vácuo, sem aditivo e estéril; centrifugadas durante 5 minutos a $720 \mathrm{xg}$ e então era realizada a separação de plasma e células da série vermelha. 0 plasma foi mantido em tubos Eppendorf ${ }^{\circledR}$ e congelado em freezer doméstico a partir do dia em que cada amostra era coletada até o dia em que todas as amostras foram processadas. As análises bioquímicas do plasma foram realizadas no Laboratório Escola da Faculdade Patos de Minas (FPM), 80 dias após a primeira coleta (correspondendo assim ao tempo máximo de congelamento das amostras). Foram dosadas as concentrações plasmáticas de Cálcio, Fósforo e Ácido Úrico de todos os exemplares. Estas análises foram realizadas em analisador automático FLEXOR XL (Vital scientific/Elitch), calibrado com calibrador multiparamétrico (Elitech Elical II) e aferido com soro controle (Control Lab). Foram utilizados reagentes da marca Elitech Clinical Systems SAS, à temperatura de $37^{\circ} \mathrm{C}$. A metodologia empregada para determinação do cálcio foi o Arsenazo III, para o fósforo, Molibdato UV e para o Ácido Úrico, enzimático colorimétrico (uricase/peroxidase).

Cálculo estatístico. (significância p<0,05) Os dados foram catalogados com auxílio do programa Microsoft Office Excel 2007 e as análises estatísticas foram processadas com o programa Bioestat 5.0. Foi realizado o teste Kolmogorov-Smirnov para normalidade, então o Teste T para duas amostras em dados considerados normais. Para os parâmetros frequência cardíaca e reflexo postural de endireitamento foram realizados testes de regressão. Os parâmetros bioquímicos foram comparados utilizando o teste ANOVA: um critério. Para os parâmetros subjetivos foi realizada análise de variância com os testes de Kruskal-Wallis e de Dunn.

\section{RESULTADOS E DISCUSSÃO}

\section{Animais e influência da temperatura}

A temperatura cloacal no tempo 0 (zero), antes da administração do fármaco era em média de $29,29 \pm 0,72^{\circ} \mathrm{C}$ e no final do experimento de $29.45 \pm 0,77^{\circ} \mathrm{C}$; não havendo diferença significativa entre as temperaturas aferidas nos dois tempos ( $\mathrm{t}=-0,3172, \mathrm{p}=0,7538)$. A temperatura ambiente mínima registrada durante o experimento foi de $25^{\circ} \mathrm{C}$, a máxima $30^{\circ} \mathrm{C}$, sendo a temperatura média $27,46 \pm 1,91^{\circ} \mathrm{C}$. A zona de temperatura considerada ótima para a espécie Boa constrictor está entre 28 e $30^{\circ} \mathrm{C}$, sendo tolerado aumento de até $5^{\circ} \mathrm{C}$ para aquecimento (i.e. banho de sol) e diminuição de até $5^{\circ} \mathrm{C}$ à noite (Divers 1996). As temperaturas às quais as jiboias foram submetidas durante o experimento são toleradas pela espécie e em média encontram-se próximas ao intervalo de temperatura ótima.

\section{Monitoramento da frequência cardíaca}

A média da frequência cardíaca (FC) seguiu a tendência ilustrada na Figura 1. Houve diferença significativa $(p=0,0049)$ entre a frequência cardíaca antes da sedação e após o efeito do fármaco. Este parâmetro foi avaliado durante o trabalho, não 


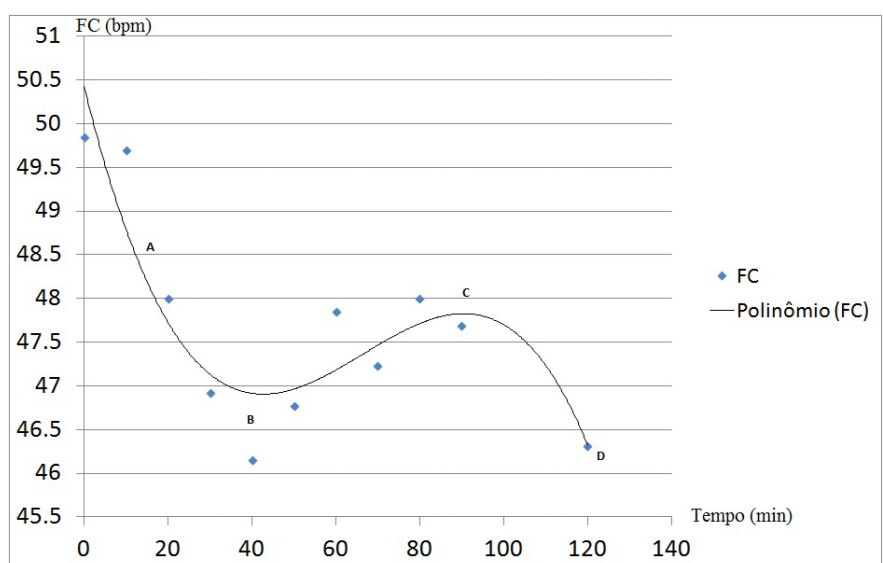

Fig.1. Frequência Cardíaca. Médias de frequência cardíaca (FC) em batimentos por minuto (bpm) das jiboias submetidas à cetamina pela via cólon-retal, em função do tempo em minutos (min), sendo o tempo 0 (zero) o momento antes da administração do fármaco e 120 o momento da última aferição. 0 gráfico evidencia uma redução na FC média a partir dos 10 minutos de administração do fármaco (A), atingindo um valor mínimo aos 40 minutos (B) então aumentando novamente até os 90 minutos de observação (C). Observa-se no tempo de 120 minutos um novo decréscimo (D). Foi realizado teste de regressão polinomial cúbica e o gráfico de dispersão segue a função $y=2 E-05 x^{3}+0,0034 x^{2}-0,1958 x+50,425$ com $\mathrm{R}^{2}=0,8319$.

apenas como um sinal da ação do fármaco, mas, principalmente, como forma de monitorar a função vital dos animais utilizados neste estudo e garantir a segurança do procedimento, para os mesmos.

O monitoramento cardíaco durante o efeito anestésico pode ser feito por palpação e auscultação com estetoscópio e com auxílio de doppler ultrassônico, menos utilizados e também descritos estão os métodos de eletrocardiograma e a oximetria de pulso (Murray 2006). Este monitoramento torna-se ainda mais importante por causa da tendência dos répteis em exibir apneia prolongada e na capacidade de manter-se em respiração anaeróbica por um longo período, complicando a manutenção de adequada função cardiorrespiratória (Murray 2006). Isto também justifica a ausência do monitoramento da função respiratória neste trabalho, pois diante de todas as causas fisiológicas que alteram esta função, não seria possível identificar a influência do fármaco na mesma.

Carregaro et al. (2009) encontraram grande variação na frequência cardíaca de cascavéis submetidas a anestesia com $80 \mathrm{mg} / \mathrm{kg}$ de cetamina pela via intramuscular em função da temperatura e evidenciaram uma diminuição na frequência relacionada à administração do fármaco, em discordância com estudos anteriores que haviam verificado aumento na FC em outras espécies de serpentes submetidas à cetamina também pela via intramuscular (Schumacher et al. 1997). Considera-se que doses de cetamina acima de $110 \mathrm{mg} / \mathrm{kg}$ produzem bradicardia a partir dos 20 minutos (Glenn et al. 1972, Bennett 1991). Apesar de utilizar uma dose menor, o presente trabalho evidenciou a mesma tendência à redução de FC, como esperado para serpentes sob o efeito de cetamina; principalmente até os primeiros 40 minutos após a administração do fármaco, quando nota-se um valor mínimo na sua média.
Nos répteis a frequência cardíaca também varia de acordo com a temperatura corporal (Murray 2006). Sugere-se que o intervalo de 30 minutos entre a penúltima e última avaliação, no qual as serpentes permaneciam em repouso e sem manipulação, justifique o novo decréscimo desta média aos 120 minutos.

\section{Parâmetros subjetivos do monitoramento anestésico}

Entre os parâmetros de avaliação do efeito anestésico, os principais citados na literatura de referência são os subjetivos (Bennett 1991, Malley 1997, Simone \& Santos 2011, Simone 2011). Neste trabalho foram avaliados a facilidade de manipulação e o relaxamento muscular concomitantemente com a mobilidade. Para possibilitar análises estatísticas e a apresentação dos resultados observados, foram atribuídos escore 1, 2 ou 3, para cada uma das serpentes em cada momento de avaliação, sendo 1 o seu estado inicial, 2 intermediário e 3 a manifestação máxima do parâmetro em questão.

Sete serpentes não apresentaram, em nenhum tempo, escore máximo para relaxamento muscular e mobilidade, tendo uma dessas permanecido em escore mínimo (1) durante todo o experimento. As demais seis serpentes permaneceram pelo menos 20 minutos em estado máximo de relaxamento. Quatro jiboias não retornaram ao seu estágio inicial de relaxamento e mobilidade dentro dos 120 minutos de observação. 0 comportamento das serpentes a respeito do critério relaxamento muscular e mobilidade está ilustrado na Figura 2a. A análise de variância identificou diferenças significativas $(\mathrm{p}<0,05)$ entre o estado inicial dos animais e o relaxamento e mobilidade nos primeiros 60 minutos após a administração do fármaco, evidenciando influência do fármaco no relaxamento muscular e mobilidade das jiboias.

Quatro animais não atingiram o escore máximo de facilidade de manipulação, sendo que um deles permaneceu durante todo o experimento em estágio de maior dificuldade de contenção. Três serpentes permaneceram em estado máximo de facilidade de manipulação a partir dos dez minutos de observação até a última observação. A tendência a aumentar a facilidade de contenção causada pela cetamina está representada na Figura 2 b. A análise de variância demonstrou que houve diferença significativa $(\mathrm{p}<0,05)$ entre o estado inicial das serpentes e os primeiros 50 minutos após a administração da cetamina. Corroborando com estudos prévios, a administração da cetamina neste experimento provocou o aumento da facilidade de manipulação das serpentes.

\section{Reação postural de endireitamento (RPE)}

A perda da reação postural de endireitamento é o sinal mais claro de que a serpente encontra-se sob o efeito do sedativo e neste trabalho todas as jiboias apresentaram algum atraso nesta reação após a administração do fármaco. Sempre que uma jibóia levava mais que 60 segundos para retomar o posicionamento natural, esta recebia auxílio do manipulador e considerava-se perda completa do reflexo de endireitamento. Isso ocorreu com sete animais em pelo menos um tempo de avaliação, sendo que um deles não voltou a apresentar o reflexo durante os 120 minutos avaliados. 0 gráfico da regressão polinomial quadrática mostra uma tendência de as serpentes levarem mais tempo para retornar ao posicionamento natural entre 10 e 20 minutos após a administração do fármaco e progressivamente diminuirem este tempo até o último momento de observação (Fig.3).

Nos estudos de Simone \& Santos (2011), as jiboias perderam a reação de endireitamento em média 15 minutos após a 


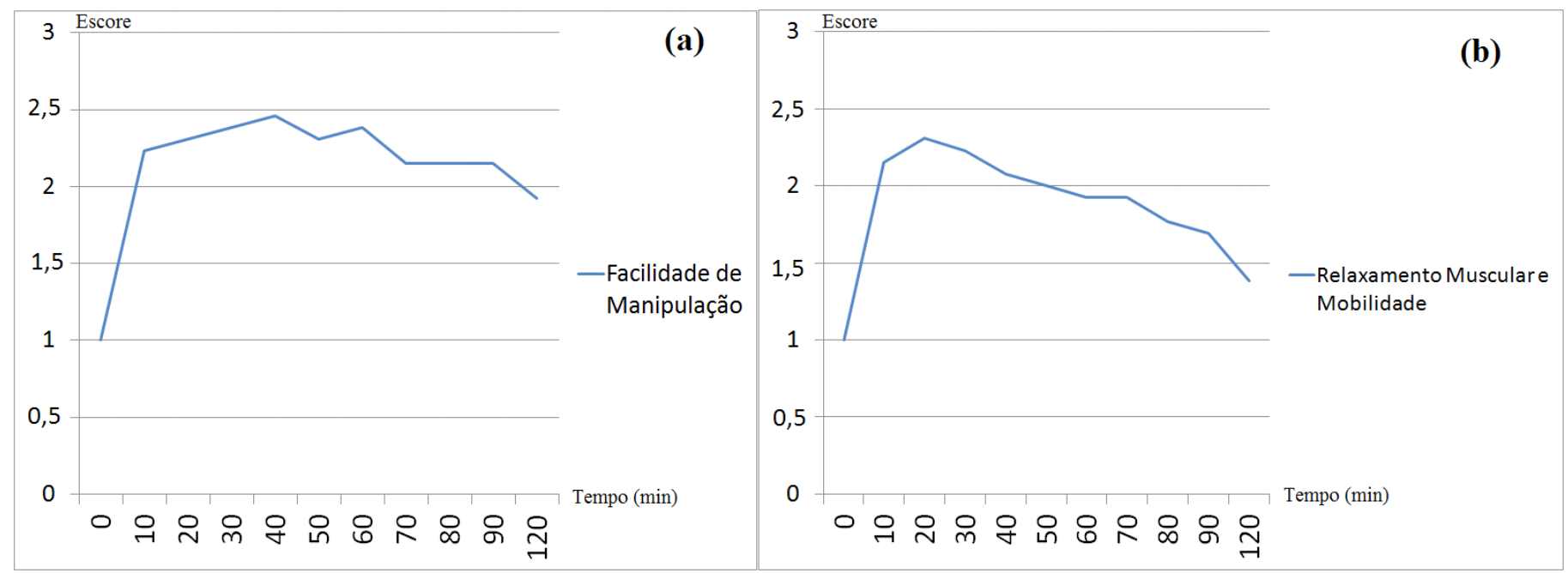

Fig.2. Parâmetros subjetivos. (a) Média para os parâmetros relaxamento muscular e mobilidade apresentados pelas jiboias durante o experimento. Quanto maior o escore maior o relaxamento e menor a mobilidade. Nota-se que a partir dos 10 primeiros minutos há um aumento do relaxamento muscular e redução da mobilidade (A), que começam a retornar ao estado inicial nos momentos finais da observação (B). (b) Média do escore de Facilidade de Manipulação das serpentes sob o efeito da cetamina. Quanto maior o escore, maior a facilidade de manipulação. Nota-se que a partir dos 10 primeiros minutos há um aumento da facilidade de manipulação (A), que começa a retornar ao estado inicial nos momentos finais da observação (B).

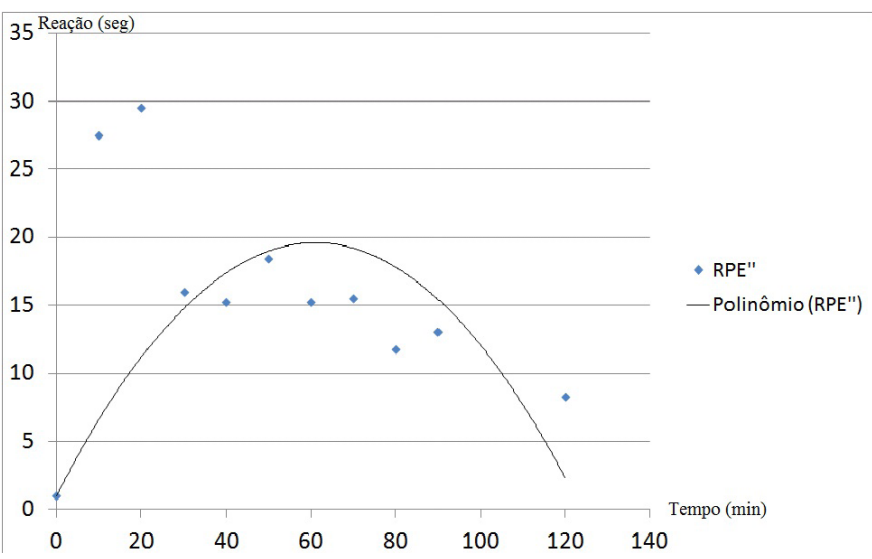

Fig.3. Reação postural de endireitamento. Gráfico de dispersão com as médias do tempo de retomada da reação postural de endireitamento, em segundos (seg) em função do tempo de monitoramento de sedação em minutos (min). Quanto maior a duração em segundos, maior a influência do anestésico sobre o animal, pois significa maior tempo de reação.

administração de $40 \mathrm{mg} / \mathrm{kg}$ de cetamina pela via intramuscular, semelhante ao ocorrido no presente trabalho, apesar de serem doses e vias de administração diferentes. Carregaro et al. (2009) em seu experimento com $80 \mathrm{mg} / \mathrm{kg}$ de cetamina, administrado pela via intramuscular em cascavéis, notaram que a reação de endireitamento foi abolida antes nos animais em temperatura de $30^{\circ} \mathrm{C}$ do que naqueles submetidos à temperatura de $25^{\circ} \mathrm{C}$. Não se pode considerar que o animal tenha se recuperado de uma anestesia geral até que ele retome a reação de endireitamento (Malley 1997), assim, no presente trabalho, nem todas as serpentes se recuperaram do efeito anestésico dentro do tempo de observação (120 minutos), apresentando no último momento registrado uma média de 8,31 segundos de duração para retomar ao seu posicionamento natural.
Outros autores também utilizaram os parâmetros, reação postural de endireitamento, frequência cardíaca, relaxamento muscular, mobilidade e facilidade de manipulação ou semelhantes para avaliação de serpentes sob efeito anestésico e em todos esses trabalhos a reação postural de endireitamento é avaliado como um dos principais indicativos do efeito anestésico (Bennett 1991, Malley 1997, Simone \& Santos 2011, Simone 2011). Neste trabalho, é possível afirmar que as serpentes apresentaram efeito sedativo após a administração de cloridrato de cetamina pela via cólon-retal, resultando em uma contenção química semelhante àquela promovida por diferentes protocolos (Bennett 1991, Malley 1997, Simone \& Santos 2011, Simone 2011).

\section{Sistema porta renal e avaliação do perfil bioquímico}

As serpentes, assim como os demais répteis apresentam o sistema porta renal, e, por isso, evita-se aplicação de fármacos na porção posterior do corpo presumindo-se o risco de fármacos com potencial nefrotóxico causarem lesão renal (Grego et al. 2014). Apesar disso, outros autores já haviam relatado o uso de cetamina injetada na porção posterior do corpo de serpentes e lagartos sem efeitos adversos e com mínima alteração na farmacocinética (Font \& Schwartz 1989, Schumacher \& Yelen 2006). Para avaliar se a administração da cetamina pela via cólon-retal submeteria o animal ao risco de lesões, foram realizadas análises bioquímicas dos indicadores da função renal.

Em répteis o aumento da ureia e da creatinina sérica não está correlacionado à insuficiência renal, assim sendo, ácido úrico, fósforo e ocasionalmente cálcio tem sido mais úteis como indicadores nesses casos (Miller 1998). O perfil bioquímico renal das serpentes foi traçado aferindo-se os valores plasmáticos de cálcio, fósforo e ácido úrico, no momento antes da administração da cetamina (Dia 1), no dia seguinte ao procedimento (Dia 2) e nove dias após (Dia 9). As médias para estes parâmetros estão indicados no Quadro 1. 
Valores elevados de fósforo constituem o indicador mais confiável de insuficiência renal (Miller 1998). Valores séricos elevados de ácido úrico podem indicar doença renal extensa, alimentação hiperproteica ou desidratação (Miller 1998). Não houve diferença significativa nos valores do primeiro, segundo e o nono dias de coleta para cálcio ( $\mathrm{p}=0,0787)$, fósforo $(\mathrm{p}=0,8999)$ e ácido úrico ( $\mathrm{p}=0,5689)$. Assim, pode-se considerar que a administração de cetamina pela via cólon-retal não provocou alterações na função renal.

A relação cálcio:fósforo sérica é utilizada para averiguar a função renal de tal forma que níveis baixos de cálcio, juntamente com níveis altos de fósforo podem indicar uma lesão. Esta relação é de aproximadamente 1,5:1 a 2:1 em serpentes saudáveis e menor nas que apresentam problemas renais (Mitchell 2009). Nenhum animal apresentou esta relação mais baixa em mais de uma aferição e apenas um apresentou valores decrescentes neste parâmetro. Não houveram alterações significativas na relação cálcio:fósforo durante o período avaliado $(\mathrm{p}=0,9855)$. Assim sendo, não foram encontradas alterações na bioquímica sérica destes animais que indiquem alteração causada pela administração da cetamina pela via cólon-retal, ao menos nos nove primeiros dias após a sua administração (Quadro 2).

\section{Protocolo anestésico e vias de administração}

A cetamina é o anestésico injetável mais comumente utilizado em répteis, podendo ser administrado pela via intramuscular ou endovenosa (Schumacher \& Yelen 2006). 0 uso do mesmo anestésico pela via retal já havia sido testado em crianças na forma de supositórios, apresentando boa analgesia, porém sem anestesia satisfatória (Pedraz et al. 1989) e em gatos domésticos, comparando-se a farmacocinética entre a administração via intramuscular, endovenosa e retal e notando efeito sedativo em todas essas vias, com taxa de eliminação semelhante e, quando utilizada a via retal, mínimo efeito de primeira passagem pelo fígado (Hanna et al. 1988). A via retal já foi testada em répteis no experimento de Wimsatt et al. (2008) para administração de claritromicina em tartarugas-do-deserto (Gopherus agassizii). Este é o primeiro trabalho a testar a via cólon-retal para administração de anestésicos em jiboias.

Apesar de não ser recomendado o uso de somente cetamina para anestesia anterior a procedimentos invasivos em serpentes, principalmente pela permanência de reflexos espinhais que podem indicar dor e pela letalidade em altas dosagens (Font \& Schwartz 1989), o presente trabalho evidenciou um protocolo seguro para a contenção química de Boa constrictor, que poderia ser utilizado para realização de exame clínico de forma mais segura e tranquila, além de procedimentos rápidos, menos invasivos e que não impliquem em dor. A dose utilizada encontra-se entre as consideradas adequadas para contenção química por outros autores que citam intervalos entre 40-80mg/kg (Glenn et al. 1972, Green et al. 1981, Hill \& Mackessy 1997) para realização de pequenos procedimentos.

Para administração de anestésicos em serpentes, a via intramuscular aparece como a mais efetiva e prática porque a via oral não é segura para répteis, a via subcutânea resulta em tempos de indução prolongados e inseguros e a via endovenosa requer dissecação (Schumacher \& Yelen 2006). Neste caso, o único sítio apropriado para administração destes fármacos em serpentes seria a musculatura paravertebral (Schumacher \& Yelen 2006). Na experimentação, a administração do fármaco
Quadro 1. Perfil bioquímico renal. Média dos parâmetros cálcio, fósforo e ácido úrico das jiboias, mensurados antes da administração da cetamina via cólon-retal (Dia 1), no dia seguinte (Dia 2) e nove dias após (Dia 9) e análise da variância que demonstrou não haver diferença significativa $(p>0,05)$ entre os três dias de aferição para todos os parâmetros

\begin{tabular}{ccccc}
\hline Parâmetros & Dia 1 & Dia 2 & Dia 9 & p valor \\
\hline Cálcio & $8,85 \pm 0,75$ & $8,07 \pm 1,29$ & $8,86 \pm 0,87$ & 0,0787 \\
Fósforo & $4,16 \pm 2,16$ & $4,34 \pm 3,15$ & $3,91 \pm 1,44$ & 0,8999 \\
Ácido Úrico & $3,38 \pm 1,15$ & $2,96 \pm 0,90$ & $3,34 \pm 1,18$ & 0,5689
\end{tabular}

Quadro 2. Relação cálcio:fósforo das treze serpentes antes (Dia 1), um dia (Dia 2) e nove dias após (Dia 9) receberem cetamina pela via cólon-retal, com as respecitivas médias e análise de variância que indicou diferença não significativa nos dias testados

\begin{tabular}{ccccc}
\hline Serpentes & Dia 1 & Dia 2 & Dia 9 & \\
\hline 1 & 3,12 & 3,22 & 1,44 & \\
2 & 1,63 & 4,63 & 2,68 & \\
3 & 1,91 & 4,04 & 3,84 & \\
4 & 3,03 & 1,13 & 2,83 & \\
5 & 0,95 & 2,41 & 2,77 & \\
6 & 1,28 & 3,83 & 3,03 & \\
7 & 2,93 & 2,09 & 1,32 & \\
8 & 2,14 & 2,38 & 1,91 & \\
9 & 3,91 & 0,88 & 1,88 & \\
10 & 2,75 & 3,14 & 3,57 & \\
11 & 2,74 & 1,52 & 2,89 & \\
12 & 2,65 & 3,09 & 2,29 & \\
13 & 3,37 & 0,87 & 2,11 & \\
Média & $2,49 \pm 0,86$ & $2,56 \pm 1,23$ & $2,50 \pm 0,76$ & $\mathrm{p}=0,9855$
\end{tabular}

foi de fácil execução, não exigindo materiais sofisticados ou técnicas complexas, revelou-se minimamente invasiva e não foram evidenciados efeitos adversos à saúde dos animais testados, além de atender às expectativas em relação aos efeitos do fármaco.

\section{CONCLUSÕES}

Foi possível promover a contenção química das jiboias Boa constrictor, utilizando $70 \mathrm{mg} / \mathrm{kg}$ de cloridrato de cetamina pela via cólon-retal de forma efetiva, menos invasiva e de fácil execução em comparação às vias convencionais.

O sistema porta renal não foi obstáculo para execução do protocolo em questão, posto que os testes bioquímicos dos níveis de cálcio, fósforo e ácido úrico demonstram ausência de lesão renal seguinte à administração do fármaco.

Agradecimentos.- Ao Hospital Veterinário da UFU. À M.V. Lilja Fromme, à Drª Líria Hirano e ao Dr Cláudio Yudi pelas contribuições.

\section{REFERÊNCIAS}

Bennett R.A. 1991. A review of anesthesia and chemical restraint in reptiles. J. Zoo Wildl. Med. 22:282-303.

Berkó S., Regdon Jr G., Ducza E., Falkay G. \& Erós I. 2002. In vitro and in vivo study in rats of rectal suppositories containing furosemida. Eur. J. Pharm. 
Biopharm. 53(3):311-315. PMid:11976019. http://dx.doi.org/10.1016/ S0939-6411(02)00005-X.

Carregaro A.B., Cruz M.L., Cherubini A.L. \& Luna S.P.L. 2009. Influência da temperatura corporal de cascavéis (Crotalus durissus) submetidas à anestesia com cetamina. Pesq. Vet. Bras. 29(12):969-973. http://dx.doi. org/10.1590/S0100-736X2009001200003.

Divers S. 1996. Basic reptile husbandry, history taking and clinical examination. J Vet Postgrad Clin Study 18(2):51-65.

Font E. \& Schwartz J.M. 1989. Ketamine as an anesthetic for some squamate reptiles. Copeia 2(2):484-486. http://dx.doi.org/10.2307/1445448.

Funk R.S. 2006. Snakes, p.42-58. In: Mader D.R. (Ed.), Reptile Medicine and Surgery. 2nd ed. Elsevier Inc., Philadelphia.

Glenn J.L., Straight R. \& Snyder C.C. 1972. Clinical use of ketamine hydrochloride as an anesthetic agent for snakes. Am. J. Vet. Res. 33(9):1901-1903. PMid:5053172.

Green C.J., Knight J., Precious S. \& Simpkin S. 1981. Ketamine alone and combined with diazepam or xylazine in laboratory animals: a 10 year experience. Lab. Anim. 15(2):163-170. PMid:7278122. http://dx.doi.or $\mathrm{g} / 10.1258 / 002367781780959107$.

Grego K.F., Albuquerque L.R. \& Kolesnikovas C.K.M. 2014. Squamata (Serpentes), p.186-218. In: Cubas Z.S., Silva J.C.R. \& Catão-Dias J.L. (Eds), Tratados de Animais Selvagens. 2ª ed. Roca, São Paulo.

Hanna R.M., Borchard R.E. \& Schmidt S.L. 1988. Pharmacokinetics of ketamine $\mathrm{HCl}$ and metabolite I in the cat: a comparison of IV, IM, and rectal administration. J. Vet. Pharmacol. Ther. 11(1):84-93. PMid:3379668. http:// dx.doi.org/10.1111/j.1365-2885.1988.tb00125.x.

Hill R.E. \& Mackessy S.P. 1997. Venom yields from several species of colubrid snakes and differential effects of ketamine. Toxicon 35(5):671-678. PMid:9203291. http://dx.doi.org/10.1016/S0041-0101(96)00174-2.

Kohrs R. \& Durieux M.E. 1998. Ketamine: teaching an old drug new tricks. Anesth. Analg. 87(5):1186-1193. PMid:9806706.

Malley D. 1997. Reptile anaesthesia and the practicing veterinarian. Clin. Pract. 19:351-368.

Massone F. 2011. Anestesia dissociativa, p.73-84. In: Ibid. (Ed.), Anestesiologia Veterinária. 6⿳亠 e ed. Guanabara Koogan, Rio de Janeiro.

Miller H. 1998. Urinary diseases of reptiles: pathophysiology and diagnosis. Semin. Avian Exot. Pet Med. 7(2):93-103. http://dx.doi.org/10.1016/ S1055-937X(98)80048-0.

Mitchell M.A. 2006. Therapeutics, p.631-664. In: Mader D.R. (Ed.), Reptile Medicine and Surgery. 2nd ed. Elsevier, Philadelphia.

Mitchell M.A. 2009. Snakes, p.136-163. In: Mitchell M.A. (Ed.), Manual of Exotic Pet Practice. Elsevier Saunders, Philadelphia.

Murray M.J. 2006. Cardiopulmonary anatomy and physiology, p.124-134. In: Mader D.R. (Ed.), Reptile Medicine and Surgery. 2nd ed. Elsevier, Philadelphia.
Oliveira C.M.B., Sakata R.K., Issy A.M. \& Garcia J.B.S. 2004. Cetamina e analgesia preemptiva. Revta Bras. Anestesiol. 54(5):739-752. PMid:19471784.

Papich M.G. 2003. Old drugs and new thoughts in exotic medicine. Comp. Cont. Educ. Pract. 25(3):5-14.

Pedraz J.L., Calvo M.B., Lanao J.M., Muriel C., Lamas J.S. \& Dominguez-Gil A. 1989. Pharmacokinetics of rectal ketamine in children. Br. J. Anaesth. 63(6):671-674. PMid:2611068. http://dx.doi.org/10.1093/bja/63.6.671.

Podell M., Wagner S.O. \& Sams R.A. 1998. Lorazepam concentrations in plasma following its intravenous and rectal administration in dogs. J. Vet. Pharmacol. Ther. 21(2):158-160. PMid:9597655. http://dx.doi. org/10.1046/j.1365-2885.1998.00117.x.

Schumacher J., Lillywhite H.B., Norman W.M. \& Jacobson E.R. 1997. Effects of ketamine HCL on cardiopulmonary function in snakes. Copeia 1997(2):395400. http://dx.doi.org/10.2307/1447760.

Schumacher J. \& Yelen T. 2006. Anesthesia and analgesia, p.442-452. In: Mader D.R. (Ed.), Reptile Medicine and Surgery. 2nd ed. Elsevier, Philadelphia.

Simone S.B.S. 2011. Avaliação dos efeitos da cetamina racêmica, cetamina s (+) e midazolam em jibóias Boa constrictor Linnaeus, 1758 (Squamata: Boidae). Dissertação de Mestrado, Universidade Federal de Uberlândia, Uberlândia, MG. 73p.

Simone S.B.S. \& Santos A.L. 2011. Efeitos da associação maleato de midazolam, citrato de fentanila e cloridrato de cetamina em jibóias Boa constrictor Linnaeus, 1758 (Squamata: Boidae). Pubvet 5(21):1132. http://dx.doi. org/10.22256/pubvet.v5n21.1132.

Steinman A., Gips M., Lavy E., Sinay I. \& Soback S. 2000. Pharmacokinetics of metronidazole in horses after intravenous, rectal and oral administration. J. Vet. Pharmacol. Ther. 23(6):353-357. PMid:11168912. http://dx.doi. org/10.1046/j.1365-2885.2000.00294.x.

Valente F.S., Bianchi S.P. \& Contesini E.A. 2013. Particularidades na contenção química e na anestesia de serpentes. Vet. Foco 10(2):210-221.

Wimsatt J., Tothill A., Offermann C.F., Sheehy J.G. \& Peloquin C.A. 2008. Long-term and per rectum disposition of clarithromycin in the desert tortoise (Gopherus agassizii). J. Am. Assoc. Lab. Anim. Sci. 47(4):41-45. PMid:18702450.

Wright K.M. 2006. Overview of amphibian medicine, p.941-971. In: Mader D.R. (Ed.), Reptile Medicine and Surgery. 2nd ed. Elsevier, Philadelphia.

Zempsky W.T. 1997. Alternative routes of drug administration: advantages and disadvantages (subject review). Pediatrics 100(1):143-152. PMid:9229706. http://dx.doi.org/10.1542/peds.100.1.143.

Zhu M., Maslow J.N., Mikota S.K., Isaza R., Dunker F., Riddle H. \& Peloquin C.A. 2005. Population pharmacokinetics of pyrazinamide in elephants. J. Vet. Pharmacol. Ther. 28(5):403-409. PMid:16207301. http://dx.doi. org/10.1111/j.1365-2885.2005.00670.x. 